\title{
Trayectorias de Formación en el Nivel Educativo Superior de los Profesionales de la Educación en Brasil Y Argentina (1990-2006)
}

\author{
Formation Trajectories In High Education Of The Professionals \\ Of The Education In Brazil And Argentina (1990-2006)
}

\section{Miriam Mabel Sanchez ${ }^{1}$}

Resumen:

Este trabajo discute comparativamente la formación de profesionales de la educación en el nivel superior en Brasil y Argentina, tomando como referencia el escenario actual de reformas educativas en los dos países. Este estudio es parte integrante de un proyecto de investigación que analiza la formación y trabajo de los profesionales de la educación: políticas, demandas sociales y prácticas pedagógicas. Ambos países atraviesan momentos de modificaciones y reestructuración en sus diferentes sistemas educativos (caso Argentino) y definiciones de lineamentos curriculares para la formación de profesionales de la educación (caso brasilero), manifestadas en leyes y políticas de educación. Analizamos a partir das referencias legales cuales son os locus de formación propuestos, en el nivel superior, para estos profesionales de la educación en los dos países y por otra parte buscamos analizar si estas indicaciones responden algún tipo de lógica que todavía no podemos desvelar con claridad.

\begin{abstract}
Así mismo se discute los significativos e definitivos procesos de transformación institucional en los que se inscribieron los procesos de formación profesional y sus posibles implicancias para la inserción laboral de los futuros egresados de tales instituciones educativas. Se resalta en la investigación las posibilidades legales de formación de los profesionales de la educación, la cual es amplia y variada (siendo posible realizarla según diferentes tipos de carga horaria, de modalidad, en diferentes tipos de Instituciones de nivel superior y de obtención de títulos finales) e que hacen que exista una gran diversidad de perfiles profesionales que compiten "igualitariamente" en el mercado de trabajo. Por ultimo se busca constituir un aporte para pensar las discusiones acerca de la formación de profesionales de la educación, que se expresan en las políticas públicas educativas actuales, en un contexto de diversidades de demandas sociales de educación y la inserción de estos profesionales en el mercado de trabajo.
\end{abstract}

Palabras clave: Formación de los profesionales de la educación; Educación superior; Políticas educativas publicas; Trabajo docente.

\footnotetext{
${ }^{1}$ Professora para o ensino primário/Pedagoga. Mestranda em Educação (PPGE-UFPR). miriamcuritiba@hotmail.com
} 
Abstract:

This work tries to discuss comparatively the professionals' formation of the education in the high education of Brazil and Argentina, taking the current stage of educational reforms as in both countries. This study is an integral part of a project of investigation that analyzes the formation and work of the professionals of the education: political, social demands and pedagogic practices. Both countries cross moments of modifications and restructuring in their different educational systems (in Argentina) and definitions of lineaments in curriculum framework for the education professionals' formation (Brazilian case), shown in laws and politics of education. We analyze the legal references which are you locus of formation proposed, in high education level, for this professionals of the education in two countries and on the other hand we analyze if these indications answer some type of logic that we cannot reveal with clarity yet. Likewise, the study discusses the significant and definitive processes of institutional transformation in those who inscribed the processes of professional training and possible implications for the market insertion of the futures teachers formed by those institutions. The investigation highlights the legal possibilities of formation of those professionals, which is wide and varied (being possible to realize it according to different types of hourly load, of form, in different types of institutions and securing final qualifications), and it does exist a big diversity of professional profiles that compete with "equality" on the labour market. Finally, this work constitutes a contribution to think the discussions about the professionals' formation in education field, which are expressed in the current public political educational, in a context of diversities of social demands for education and the insertion of these professionals on the labour market.

Keywords: FFormation of Professionals in Education field; High Education; Public Education Politics; Teaching Work. 
En los últimos años Brasil y Argentina - como tantos otros países de la región realizaron grandes reformas de sus sistemas educativos, con la finalidad de lograr avances en la calidad de los mismos, y como respuesta a exigencias de los "demandantes" organismos internacionales. Estos procesos de reforma, de reestructuración y privatización de "servicios educativos" tuvieron entre sus consecuencias directas, la reorganización de la formación de los profesionales de la educación. Así, ambos países verifican tendencias convergentes e divergentes, en el que se relaciona a las políticas de formación de los profesionales de la Educación durante el periodo analizado (1990-2006).

Conrelaciónalaformación de los profesionales delaeducación, las leyes sancionadas, posibilitan por medio de su flexibilidad, nuevos recorridos de formación, demandas de capacitación en servicio y diversificación de ofertas académicas de formación inicial. Muchas de estas propuestas, no siempre se caracterizan por su calidad.

Se pretende por medio de este abordaje comparado, avanzar en la comprensión de la lógica que subyace a las políticas de formación de estos profesionales en ambos países.

Se parte de la hipótesis de que tales políticas de formación profesional, introducidas por las respectivas leyes nacionales de educación en los dos países, fragmentan y diversifican la formación profesional. Así, se abre camino para la perdida de identidad profesional y calidad de formación en la medida en que se escoge a instituciones de educación superior no universitarias como ámbitos "ideales" de formación. Argentina y Brasil avanzan durante ese periodo, hacia una adaptación de los programas de estudio según los cambios en el perfil profesional requerido por el mercado de trabajo. Dentro de este contexto de flexibilización curricular, se propone la idea de articulación entre la formación inicial y el posgrado, ajustando el perfil profesional al nuevo paradigma de producción capitalista. Se dejan de lado cuestiones relativas a la identidad profesional y calidad de la formación inicial.
El trabajo se realizó por medio de un análisis bibliográfico y un análisis de las leyes nacionales de cada país, en lo referente a la formación de los profesionales de la educación.

En el caso argentino se tomo como referencia a Ley Federal de Educación n. 24.195/93, la Ley de educación Superior $n^{\circ}$. 24.521/95 y la reciente Ley Nacional de Educación n. 26.206/2006. Se consideraron autores como Lamarra (2003), Mollis (2003), Krotsh (2003) entre otros.

En el caso brasilero, se analiza la Ley de Directrices y Bases da Educación Nacional (LDB) N. 9.394/96 y lo referente a la aprobación de las Directrices Nacionales para el Curso de Pedagogía (2006). Los autores de referencia son Freitas (1999), Aguiar (1999-2006), Machado (2004-2006), Libaneo (2006) entre otros. También se toman como referencia publicaciones de la ANFOPE (Asociación Nacional por la Formación de los Profesionales de la Educación).

El presente articulo, forma parte de una investigación en proceso de desarrollo, y por lo tanto, se van planteando interrogantes, los cuales serán profundados en otra etapa, contando con el aporte de la Profesora Dra. Evelcy Monteiro Machado. El presente articulo, se organiza según casos (argentino y brasilero), durante los años 90 donde se realiza un análisis de las legislaciones de la época abordada, siempre teniendo como eje las propuestas de formación de los profesionales de la educación y su vinculación con el nivel superior de educación. En un segundo momento se discuten los rumbos adoptados por las recientes leyes y disposiciones curriculares para ese sector profesional. Finalmente se presentan algunas convergencias y divergencias entre los dos países.

\section{Argentina y Brasil en los años $\mathbf{9 0 .}$}

Por medio de la Ley de Educación Superior $n^{\circ}$ 24.521/95, en la Argentina, se diversifica la oferta de formación en el nivel superior no universitario, acompañado por una fuerte expansión del sector educativo privado en ese nivel. Esto hechos provocaron, según Krotsh, una nueva 
configuración del sistema de educación superior, que "puede ser caracterizado en materia de política universitaria como la de construcción de consenso con relación a la agenda educativa promovida por le Banco Mundial para América Latina" (2003, p.172). Tal legislación regula el sistema educativo horizontalmente en relación con los otros sectores de la educación superior y crea al mismo tiempo nuevos sectores, expandiendo el sistema hacia encima por medio de la creación de organismos intermedios entre la Universidad y el Estado. La Ley, según Krotsh (2003) es un instrumento regulador y constitutivo, en la medida en que establece nuevas reglas, nuevos modelos de organización institucional dentro del nivel superior de educación. En Argentina, el sistema educativo de nivel superior tiene una tendencia a ser binario, esta formado por la Educación Superior Universitaria (universidades) y la Educación Superior no Universitaria (institutos terciarios). Se produce de esta forma un panorama institucional caracterizado por un crecimiento institucional desordenado que generó un sistema heterogéneo y escasamente articulado al cual se le suman los desafíos provenientes de la descentralización. En este contexto, se formaran los profesionales de la educación según la Ley 24.195/93.

La formación inicial de los profesionales de la educación, según la ley 24.195/93, en el capitulo $\checkmark$ que hace referencia a la educación superior, segundos artículos 18,21 y 24 se hacen referencias puntuales sobre esta formación y los ámbitos en los cuales transcurrirá: Institutos de formación docente (grado), Universidad (grado universitario) y otras instituciones organizadas para tal fin.

Se deja abierta la posibilidad de creación de otras instancias de formación y se muestra una intencionalidad de establecer articulaciones entre las diversas instituciones. Estos hechos, ocasionan un aumento de ofertas de formación, las cuales presentan carga horaria, perfiles y calidad de formación diferentes.

La ley de Educación Superior no 24.521/95 intensifica estas propuestas de diversificación y fragmentación en la formación docente, ya manifestadas en la Ley del 93. Con la sanción de esta Ley de Educación Superior, por primera vez en la legislación argentina, se incluye conjuntamente dentro del nivel de educación superior, la educación universitaria y no universitaria. De esta forma dentro del nivel de educación superior argentino encontramos Instituciones Universitarias (universidades e Institutos universitarios), Institutos Terciarios y Colegios Universitarios.

Los institutos terciarios existen en la Argentina desde los primeros años del siglo $X X$, siendo privados o públicos, y durante mucho tiempo dependieron del Poder Central. A partir la Ley $n^{\circ}$. 24.049/92 de transferencia de los servicios educativos nacionales para las provincias, "las instituciones de Educación Superior no universitarias se transfirieron a las respectivas provincias, conjuntamente con las escuelas de nivel medio" (LAMARRA, 2003, p. 69).

La formación de los docentes para todos los niveles educativos esta históricamente a cargo de las Instituciones de Educación Superior desde la década de los 70, siendo que la mayor fracción se realiza en este tipo de instituciones.

Las carreras cursadas en el terciario no dan un titulo de grado, ya que la Ley de Educación Superior expresa en su artículo 40 que compete exclusivamente a las Instituciones Universitarias otorgar títulos de grado de licenciados y títulos profesionales equivalentes. Las instituciones no universitarias solo pueden ofertar carreras y títulos de pre-grado.

Desde la sanción de la ley de educación superior $n^{\circ} 24.521 / 95$, se van proponiendo algunas estrategias de articulación entre las diferentes instituciones que componen el sistema educativo de nivel superior, como el caso de las "licenciaturas de complementación". Tales licenciaturas, son ofrecidas por las universidades dentro de una propuesta de complementación para los egresados de los Institutos de Educación Superior o universitarios. Según Lamarra (2003), el 75\% de esos cursos de complementación corresponden a la modalidad Docente con el titulo de Profesor, lo cual posibilita la obtención del título de Licenciado en Educación. 
Las carreras terciarias o de pre-grado se validan a través del Decreto $n^{\circ}$ 1276/96 que reglamenta el régimen de funcionamiento de esas Instituciones. Las universidades también constituyen un ámbito de formación de los profesores para diferentes niveles de enseñanza (inicial y primario) y de carreras como Licenciado en Ciencias de la Educación, Profesor en Ciencias de la Educación para nivel medio y superior. La diferencia entre las carreras cursadas en los Institutos y las universidades, es que en este ultimo caso, las actividades de investigación son parte integrante de los currículos como una dimensión continua de la formación.

Según lo analizado, podemos afirmar que las leyes de este periodo, tienen una tendencia a continuar con la fragmentación y desarticulación del sistema educativo en el nivel superior, manteniendo la histórica asimetría entre el sector universitario "que concentra el $74 \%$ de la matricula total de ese nivel educativo frente las Instituciones de nivel superior no universitario que cuentan con el $26 \%$ restante de los matriculados" (LAMARRA, 2003, p. 108).

La afirmación de que la formación inicial de los profesionales de la educación sea en los Institutos de Formación Docente en el ámbito superior no universitario (Ley Federal de Educación Nº. 24.195/93), abre camino para el surgimiento de propuestas de formación diferenciada y de calidad variada, aumentando el caos y diversificación de instituciones ya existente en el conjunto del sistema educativo.

Si bien la ley de educación superior hace referencia a la posibilidad de articulación entre las Instituciones, también taxativamente estipula por medio del articulo39(determinando el tipo de título) quienes se encuentran habilitados para realizar pos-graduaciones. En el artículo 36, establece los requisitos, en materia de credenciales educativas, para el ejercicio de la docencia en el nivel superior (no puede desempeñarse como tal un egresado de una Institución no Universitaria). Estas medidas reguladoras que prohíben y limitan el campo de acción profesional de los egresados de los Institutos de Formación Docente no universitarios, denotan una clara diferenciación entre un diploma de una Universidad y un diploma de un Instituto de Formación Docente. De esta forma, claramente se refuerzan las desigualdades implícitas a los ámbitos de formación profesional.

Además de estas fallas en la articulación entre Instituciones dentro del nivel superior, se suma aquellas relacionadas con el proceso de formación de los profesionales de la educación. Existe claramente una separación entre las carreras que se cursan en los Institutos de formación Docente, ligadas a la docencia y una marcada inclinación para la investigación en la formación universitaria. Si bien, ambas actividades deberían formar parte de la formación de los profesionales de la educación, ellas ocurren de forma separada, en contextos institucionales diferentes y sin aparente ligación entre las mismas.

A estas "confusiones" originadas por la gran diversidad de denominación de títulos ofertados, se le suman las "especializaciones" que hacen con que existan profesionales de la educación que son: idóneos, experto, asistente educativo, analista-técnico, técnico superior en educación, en Ciencias de la Educación, en gestión escolar, gestión de instituciones escolares o educativas, en educación primaria... entre otros. Con relación a la carga horaria de estas posibilidades de formación, las mismas oscilan entre 1800 e 3800 hs reloj. Todos estos "títulos" compiten por un lugar en le mercado de trabajo, y son producto de un escenario de medidas neoliberales para la formación de los profesionales de la educación.

En el caso brasilero, desde el análisis de las políticas de educación superior se desprende que estas están asociadas a un proyecto neoliberal de minimización del Estado. Según Dourado (2003), se puede manifestar que las políticas de educación superior tuvieron un carácter instrumental, siendo uno de sus objetivos la reorganización institucional y la descentralización de la gestión, el fortalecimiento de la autonomía, realizar reformas curriculares y instaurar fuertemente la capacitación en servicio de los profesionales de la educación.

Esa tentativa de mejorar la calidad de la formación inicial, se plasma en la LDB cuando 
se manifiesta que la formación de Profesores deberá ocurrir en los Institutos Superiores de Educación y en los Cursos de Normal Superior. Tales instituciones son reglamentadas por el Decreto $n^{\circ} 2307 / 97$ que posibilita la diversificación de las instituciones de educación superior, y reconoce que os Institutos de Ensino Superior son solo instituciones de enseñanza, separadas de la investigación y la extensión. Esta postura desarticula instancias de formación que son indisociables entre si. Como señal Maues, "esa característica, de formación en una institución apenas de enseñanza, nos da nociones de la disminución del valor que se pasa a dar a la formación de los profesionales de la educación" (2003, p.19).

La Ley de Directrices y Bases de la Educación n. 9394/93, según Machado "[...] pone en evidencia la sumisión del gobierno a los acuerdo con el Banco Mundial y otros agentes externos, en relación a la formación de los profesores" (2006, p.126).

En este contexto, dicha Ley, en relación a los profesionales de la educación y su formación va introducir algunos cambios significativos. El contenido de tres artículos de la nueva Ley $n^{\circ}$ 9394/96 fueron los responsables por los impactos iniciales para un proceso de transformación y discusión sobre la formación de estos profesionales. Así la Ley expresa:

Art. 62. La formación de docentes para actuar en la educación básica se realizará en el nivel superior, en carrera de Licenciatura, de graduación plena, en universidades e institutos superiores de educación, admitida como formación mínima para el ejercicio de la docencia en la educación inicial y los primeros cuatro años de la enseñanza fundamental, la que se ofrece en el nivel medio, en la modalidad Normal (BRASIL, 1996).

De esta forma, el Atr. 62 introduce la figura de los Institutos Superiores de Educación para que conjuntamente con las Universidades (ambos dentro del nivel superior educativo) se constituyan en ámbitos de formación de los docentes para actuar en la educación básica. La última parte de este articulo, será modificada en el año 1999 por medio del Decreto n 3.276 , que dispone la formación en el nivel superior de los profesores que actúen en la educación básica.

El artículo 63, instituye entre los cursos a ser ofertados, el Normal Superior. Tal curso esta destinado a formar docentes para educación infantil y los primeros años de la enseñanza fundamental.

Por último el artículo 64 deja expresado las dos instancias alternativas de formación de los profesionales de la educación para las áreas de administración y gestión educativa de la enseñanza básica, siendo estos las carreras de graduación en Pedagogía o lo ofertado en nivel de pos-graduación. Podemos afirmar, que según lo expresado en el artículo 61, la identidad de esta formación esta vinculada a la docencia.

Con referencia a los Institutos Superiores de educación, tanto en Argentina como en Brasil, serán los locus de formación "privilegiados" en este periodo por las legislaciones nacionales. Estas instituciones de enseñanza, en los dos países, constituyen instancias de formación separadas de las universidades, pero dentro del nivel superior de educación. Los profesores se forman separados del resto de los profesionales de la educación.

En el caso brasilero, por medio del Decreto $n^{\circ}$ 2032/97, que modifica el sistema federal de educación superior, se permite que las instituciones de enseñaza del sistema federal se organicen académicamente en universidades, centros universitarios, facultades integradas, facultades e Institutos Superiores de Educación.

La generalidad y flexibilidad de la Ley de Directrices y Bases de la Educación, Ley $n^{\circ}$ 9.394/96, posibilitan el resurgimiento de los Institutos Superiores y la introducción del Curso Normal Superior. Con la creación de este tipo de instituciones independientes entre si, se produce una formación separada de la investigación y una perdida de la perspectiva transformadora de la educación.

Esta legislación declara que la formación de los profesionales de la educación tendrá lugar en el nivel superior del sistema educativo, no haciendo distinciones, como en le caso argentino, entre instituciones universitarias y no universitarias. 
Con esta nueva configuración del nivel superior, el campo de formación de los profesionales de la educación se amplía e diversifica. En el año 1999, por medio del Decreto Presidencial $n^{\circ} 3276 / 99$ se pretende limitar el ámbito de formación de los profesores para educación infantil y primeros años de enseñanza fundamental, cuando se dispone que "exclusivamente" el Curso de Normal Superior formara a estos profesionales. La presión de las asociaciones de profesionales de la educación provocara que el Gobierno mude tal propuesta.

Del análisis de tales políticas, en los dos países, podemos señalar que las instancias de formación para los profesionales de la educación refuerzan la tradición de descalificación de tales profesionales. Este tipo de formación fuera del ámbito universitario, viene acompañada de carreras con nominaciones diferenciadas lo cual contribuye al avance del sector privado en materia de ofertas de formación. Tal avance se ve favorecido por las "demandas de formación de profesores sin financiamiento público para instituciones públicas por medio de la expansión de instituciones privadas" (MACHADO, 2006, p.128). Estas posibilidades de formación en el Brasil al igual que en la Argentina, colocan en el mercado de trabajo una gran diversidad de profesionales con diplomas parecidos o semejantes, sin embargo, con trayectorias académicas diferentes.

En los países se produce una jerarquía dentro del nivel superior, en el cual los institutos de educación superior ocupan los últimos lugares. Se busca adaptar el nivel superior de la educación formal a las demandas del mercado y al proceso de re-configuración del Estado.

\section{Nuevos rumbos en las legislaciones del periodo 2005-2006?}

La décadadel 90 dejacuestionessinresolución en los sistemas educativos de Argentina y Brasil. La concretización de las reformas propuestas por las legislaciones nacionales enfrentó dificultades de implementación y de aceptación por parte de la comunidad educativa y los profesionales de la educación. Al mismo tiempo los dos países enfrentaron cierta coyuntura política que dificultó la realización de las metas propuestas.

Creemos que no se siguieron "nuevos rumbos" sino que más bien se definieron los caminos incipientemente delineados en el periodo anterior.

En este periodo, a partir del año 2000, los dos países van atravesar profundas crisis económicas, políticas y sociales producto de una década de neoliberalismo para los "países en desarrollo".

En el caso argentino durante esta etapa, nuevas demandas vinculadas a antiguas carencias de calidad educativa, serán discutidas y la Ley Federal de Educación del año 1993 (vigente en algunas provincias y en otras no) será reemplazada por la Ley Nacional de Educación n 26.206/2006.

Un aspecto relevante para este trabajo de investigación, esta vinculado a la creación del Instituto Nacional de Formación Docente, en el ámbito del Ministerio de Educación, Ciencia y Tecnología. Este tipo de propuesta argumenta una pretendidaidea de homogeneizary elevar la calidad de la formación de esos profesionales, por medio de un reglamentado sistema de certificaciones y evaluación de las instituciones responsables por la formación docente. La Ley n²6.206/2006 se enmarca en una "preocupación" por parte del poder central por la falta de estructura unificada del sistema educativo, para todo el país. Según palabras del Ministro Filmus, se deben trazar políticas integrales destinadas a mejorar la calidad de la formación inicial y continua de los docentes. El problema seria la ausencia de un sistema nacional de formación y pasa a considerarse esta cuestión como un tema de importancia estratégica, el cual tiene que ser abordado a nivel nacional.

La nueva ley expresa en su articulo 72 que la formación docente es parte integrante del nivel de Educación Superior y tiene entre sus funciones, la formación docente inicial, la formación docente continua, el apoyo pedagógico a las esuelas y la investigación educativa.

La carrera docente tendrá dos ciclos, una formación básica y otra especializada, siendo la carga horaria mínima de 1800 hs reloj.

Se produce una modificación con relación a las instituciones de formación de los profesionales 
de la educación, con la creación del Instituto Nacional de Formación docente como órgano responsable de planificar y ejecutar políticas de articulación del sistema de formación docente inicial y continua, promover políticas nacionales y lineamentos básicos curriculares para la formación docente inicial y continua, aplicar las regulaciones que rigen el sistema de formación docente en cuanto a evaluación, auto evaluación y acreditación de instituciones y carreras, validez nacional de títulos y certificaciones, en todo lo que no resulten de la aplicación de las disposiciones específicas referidas al nivel Universitario de la Ley № 24.521/95, entre otras cuestiones.

Con este escenario de fondo, se van a desenvolver las acciones de formación de los profesionales de la educación, fuera de las universidades, dentro de la educación superior. Esta novedad tiene un carácter centralizador que pretende regular a nivel nacional, la formación de los profesionales de la educación. Teniendo en cuenta sus atribuciones, podemos manifestar que dependerá de la voluntad política del Ministerio de Educación realmente concretizar cuestiones como la articulación con otros niveles de formación, definición de delineamentos curriculares nacionales y por sobre todo promover la investigación en el ámbito de los Institutos.

Conlaley 26.206/2006 se produceuna definición institucional significativa, en lo relacionado a las Instituciones de educación superior no universitarias, las mismas según el articulo 133 de esa ley, cambian su denominación pasando a ser denominadas "institutos de educación superior". Parecería ser que estos cambios en la nomenclatura buscan rescatar el prestigio perdido de estas instituciones de formación, pero todavía es temprano para realizar ese tipo de afirmaciones.

En Brasil, después de un largo periodo de discusiones se definen a nivel nacional las Directrices NacionalesparaelCursodePedagogía en el año 2006. Este demorado debate, brinda al curso una definición en relación a su identidad y funciones de formación. Tal carrera, se cursa en las Universidades y forma a los profesores para los nivel de enseñanza inicial, los primeros años de la enseñanza básica y a los pedagogos. Es un curso con una duración de cuatro años y una carga horaria de 3200hs mínima, a partir de las nuevas Directrices Curriculares.

Si bien se definieron las bases curriculares, se continúa debatiendo acercasi el curso solo prepara para la docencia para los niveles mencionados o si forma un tipo de especialista capacitado para ejercer tareas de gestión y administración escolar. Con la definición de estas directrices el Curso Normal Superior, pasaría a ser un curso que pude complementarse por medio de la Licenciatura en Pedagogía en las Universidades.

Vemos como Brasil todavía, se busca definir el locus de formación de sus profesionales de la educación, y en un escenario institucional mucho mas diversificado que el de Argentina. En los días actuales, existe el curso normal de nivel medio que coexiste con los cursos de nivel superior (en universidades por medio del curso de pedagogía, y los ofertados por los Institutos superiores que ofrecen el Normal Superior y el curso de Pedagogía), mientras que en la Argentina se privilegian los Institutos de Formación Docente.

\section{Palabras finales}

En los dos países durante la época de los 90 se producen reformas impuestas que llevan a la creación de modelos "alternativos" de formación, lo que produce lamentablemente una fragmentación y debilitamiento del nivel superior público. En Brasil la oferta de este tipo de cursos se da principalmente por medio del sector privado, que ve la oportunidad de captar un tipo de cliente que necesita, por demandas de la legislación, tener un diploma de una institución de nivel superior para continuar o inserirse en el mercado de trabajo. De esta forma, los antiguos cursos de normal superior, están pasando a se denominados, Cursos de Pedagogía, dando respuesta (en la mayoría de los casos, de forma solo nominal) a los requerimientos de formación explicitados en la DCN do curso de Pedagogía. En la Argentina, a partir de la creación del Instituto Nacional de Formación Docente, parecería ser que se va a dar una re-valorización a 
los tradicionales institutos terciarios o Institutos de formación docente, que como ya he mencionado históricamente formaron a los profesionales de la educación en el país.

Comparativamente vemos una demora temporal en Brasil para definir el nivel educativo en el cual se formaran los profesionales de la educación. Será recién en la década del 90 que se demanda de formación en el nivel superior para esos profesionales, este desafío era para ser alcanzado en el periodo 1996-2007, lamentablemente ese objetivo no fue alcanzado y el plazo se extendió hasta el año 2010. De la misma manera, conforme lo manifiesta la ANFOPE (Associação Nacional pela Formação dos Professionais da Educação) coexisten profesionales formados el nivel medio con otros formados en nivel superior, a lo cual se le suma los egresados de los cursos de Pedagogía a Distancia (cursos de un año y medio de duración, con encuentros mensuales y con apoyo de tutores presénciales y on-line).

Argentina se encuentra en un proceso de centralización y definición de las cuestiones de formación de sus profesionales de la educación. Parecería ser una propuesta interesante que no se abandonen las ideas y propuesta de articulación con las Universidades y que por otra parte se mantenga el desafío de incluir la investigación y la reflexión sobre las prácticas en los programas de formación inicial y continua. Brasil necesitaría rever la Educación a Distancia como modelo de formación de los profesores, evaluar la situación del Curso Normal de nivel medio para la formación de profesores para Educación Infantil y las primeras series de la Educación Fundamental (equivalente al primero a cuarto grado de la escuela primaria argentina) y encarar definitivamente el fortalecimiento de las facultades y centros de educación de las Instituciones de Educación Superior públicas, entre otras cuestiones.

Otra divergencia es que en caso argentino la formación de los profesores se va a centralizar en los Institutos de formación docente, privilegiando ese ámbito. Si bien la universidad ofrece esa posibilidad de formación, la política nacional define, entrelineas, su intención de locus de formación inicial. Estas medidas refuerzan en el caso argentino, la formación de los especialistas en las universidades y la formación de profesores en otro tipo de instituciones educativas. Contrariamente Brasil, como hemos mencionado realiza un avance importante al definir como locus de formación de sus profesores y especialistas en las en carreras de licenciatura, aunque deja librado a las instituciones oferentes el perfil y tipo de formación a desarrollar. Seria necesario que esta definiciones fueran acompañadas de rigurosos procesos de creación, acreditación y autorización de las carrera ofertadas y una evaluación permanente de los mismos por una Comisión Nacional, según lo manifestado por la ANFOPE en su último Boletín (ANFOPE, 2007).

En el interior de las convergencias encontramos que en los dos países se produce una fragmentación dentro del nivel superior de educación, que se intensifica a partir de los años 90, lo cual tras repercusiones para la formación de los profesionales de la educación; que se adoptan recomendaciones de los organismos internacionales que buscan, segundas propuestas del Banco Mundial, dar certificados habilitantes en lugar de ofrecer una formación de calidad acorde a la importancia del rol educativo. Tanto en Argentina como en Brasil, las políticas de formación definidas para los profesionales de la educación se concretizan en un nivel mas bajo e de menor costo de lo que ocurre en las Universidades.

Estas apreciaciones nos permiten concluir que existe una contradicción entre el aparente interés de los Estados argentino e brasilero por la calidad de la enseñanza básica y las políticas de formación para los profesionales de ese nivel educativo.

El avance del mercado se da por medio de la oferta privada de formación de los profesionales de la educación. Es el mercado de trabajo quien regula las instancias de formación, los diplomas pasan a tener un valor determinado según la institución que los otorga. Como describe Mollis (2003), las políticas neoliberales, la transformación del contrato social entre el Estado y la sociedad civil convirtiendo los "saberes universitarios" en 
"conocimientos mercantilizados". Las leyes de los dos países dejan abierta esa posibilidad de oferta y demanda en manos del "todopoderoso" mercado que todo lo pude, que todo lo regula.

Seria conveniente, que se buscara valorizar los diplomas y la formación por medio de una reorganización de la oferta académica educativa, ya que a diversidad de posibilidades y alternativas de formación afecta negativamente a los egresados de tales carreras, en lo relativo a su inserción laboral.

Si se continúa con esta lógica de diversificación, se destruye la posibilidad de construcción de la identidad del profesional de la educación, lo que contribuye a "justificar" los bajos salarios, las condiciones precarias de trabajo y la desvalorización social del docente.

Bibliografia

ARGENTINA/MEC. Lei n. 24.521,20 de Julio de 1995. Ley de Educación Superior, Buenos Aires, Boletín oficial $n^{\circ} .2820$, publicada 10 de Agosto de 1995. Disponível em: www.me.gov.ar/consejo/cf_ley superior

Lei 26.206, Lei de Educación Nacional. Disponível em www. mec.gov.ar/doc_pdf/

ley_educ_nac_pdf.

BORTHWICK, M. Legislación educativa argentina, Ley federal de Educación n²4.195/93. Buenos

Aires: Ripari, 1995.

BRASIL. Lei $\mathbf{n}^{\circ}$. 9.394, de 20 de Dezembro de 1996. Estabelece as Diretrizes e bases da educação

nacional. Diário Oficial da União. Brasília: DF, 23 dez. 1996.

Parecer CNE/CP n. 5/2005. Diretrizes Nacionais para o Curso de Pedagogia. Aprovado em 13/12/2005.

DOURADO, L.; CATANI, A. M; OLIVEIRA, J. F. Políticas e gestão da educação superior:

transformações recentes e debates atuais. São Paulo: Alternativa, 2003.

KROTSCH, P. Educación superior y reformas comparadas. 1. ed. Provincia de Buenos Aires:

Universidad Nacional de Quilmes, 2003.

LAMARRA, N. La educación superior en debate. Buenos Aires: Eudeba, 2003.

MACHADO, E. M. Políticas para a formação de professores no contexto neoliberal. En: MACHADO, E M y MUELLER, H.I. (Org.) Políticas Públicas Múltiplos olhares. ljuí: Unijuí, 2006.

MAUÉS, O. Os organismos internacionais e as políticas públicas educacionais no Brasil. En:

GONÇALVES OLIVEIRA, Luiz Alberto (Org). Currículo e políticas públicas. Belo Horizonte: Autêntica, 2003.

MOLLIS, M. Un breve diagnostico de las universidades argentinas: identidades alteradas. MOLLIS M. (Org.) In: Las universidades en América Latina: Reformadas o Alteradas? la cosmética del poder financiero. Buenos Aires: CLACSO, 2003.

Recebido em junho de 2008

Aprovado em junho de 2008 